\title{
Positional posterior plagiocephaly: a single-center review
}

\author{
Ranbir Ahluwalia, BS, ${ }^{1,2}$ Chelsea Kiely, ${ }^{1,3}$ Jarrett Foster, BS, ${ }^{1,4}$ Stephen Gannon, CCRP, ${ }^{1}$ \\ Alyssa L. Wiseman, MA, MS, ${ }^{1}$ Chevis N. Shannon, DrPH, MBA, ${ }^{1,5}$ and \\ Christopher M. Bonfield, MD1,5
}

\begin{abstract}
${ }^{1}$ Surgical Outcomes Center for Kids, Monroe Carell Jr. Children's Hospital at Vanderbilt, Nashville, Tennessee; ${ }^{2}$ Florida State University College of Medicine, Tallahassee, Florida; ${ }^{3}$ Cornell University Department of Neurobiology, Ithaca, New York; ${ }^{4}$ University of South Carolina School of Medicine, Columbia, South Carolina; and ${ }^{5}$ Department of Neurological Surgery, Vanderbilt University Medical Center, Nashville, Tennessee
\end{abstract}

\begin{abstract}
OBJECTIVE The authors sought to assess the prevalence and severity of positional posterior plagiocephaly (PPP) in the pediatric population at a tertiary care center.

METHODS The authors conducted a retrospective review of 1429 consecutive patients aged 2 months to 18 years who presented with head trauma and a negative CT scan in 2018. The cohort was stratified by age. The cranial vault asymmetry index (CVAl) was calculated at the superior orbital rim. Asymmetry was categorized according to the following CVAI scores: mild $(3.5 \%-7 \%)$, moderate $(7 \%-12 \%)$, and severe $(>12 \%)$. Patients were grouped by age to assess PPP at different stages of head development: group 1, 2-5 months; group 2, 6-11 months; group 3, 12-23 months; group 4: 2-4 years; group 5, 5-8 years; group 6, 9-12 years; and group 7, 13-18 years. Patients with a history of shunted hydrocephalus, craniosynostosis, skull surgery, or radiographic evidence of intracranial trauma were excluded.
\end{abstract}

RESULTS The overall cohort prevalence of PPP was 24.8\% (354 patients). PPP prevalence was higher among younger patients from groups $1-3(40.4 \%, 33.5 \%$, and $0.8 \%$, respectively). There was a continued decline in PPP by age in groups $4-7$ (26.4\%, 20\%, 20\%, and $10.8 \%$, respectively). Mild cranial vault asymmetry was noted most often $(78.0 \%$, 276 patients), followed by moderate (19.5\%, 69 patients) and severe $(2.5 \%, 9$ patients). There were no patients in group 6 or 7 with severe PPP.

CONCLUSIONS PPP is prevalent in pediatric populations and most commonly presents as a case of mild asymmetry. Although there was an overall decline of PPP prevalence with increasing age, moderate asymmetry was seen in all age groups. No patients in the cohort had severe asymmetry that persisted into adolescence.

https://thejns.org/doi/abs/10.3171/2019.12.PEDS19651

KEYWORDS craniofacial; plagiocephaly; cranial vault asymmetry index; "Back to Sleep"

$\mathrm{P}$ OSITIONAL posterior plagiocephaly (PPP) is defined as nonsynostotic flattening of the back of the skull with associated facial asymmetry. ${ }^{11}$ The flattening comes from external forces pushing on the head that can result from the positioning of an infant during sleep and play. ${ }^{10}$ Previously reported studies have shown that plagiocephaly is not strictly a cosmetic condition; in rare cases it can be associated with delays in mental, motor, and language development. ${ }^{9,11}$ While first-line treatments include repositioning, physical therapy, and "tummy time," helmet therapy is also employed in certain patients. After a child's first year, treatment for positional plagiocephaly becomes less effective and longer in duration, making early and accurate diagnosis crucial. ${ }^{5}$

The prevalence of plagiocephaly has been difficult to assess since the American Academy of Pediatrics (AAP) released the Back to Sleep campaign in 1992 as an effort to decrease the incidence of sudden infant death syndrome (SIDS). This effort resulted in the recommendation that children should sleep in the supine rather than the prone position. ${ }^{10}$ As the supine position was adopted, a subsequent lower rate of SIDS was followed by a rise in posi-

ABBREVIATIONS AAP = American Academy of Pediatrics; CVAI = cranial vault asymmetry index; MCJCHV = Monroe Carell Jr. Children's Hospital at Vanderbilt; PPP = positional posterior plagiocephaly.

SUBMITTED October 28, 2019. ACCEPTED December 5, 2019.

INCLUDE WHEN CITING Published online January 31, 2020; DOI: 10.3171/2019.12.PEDS19651. 


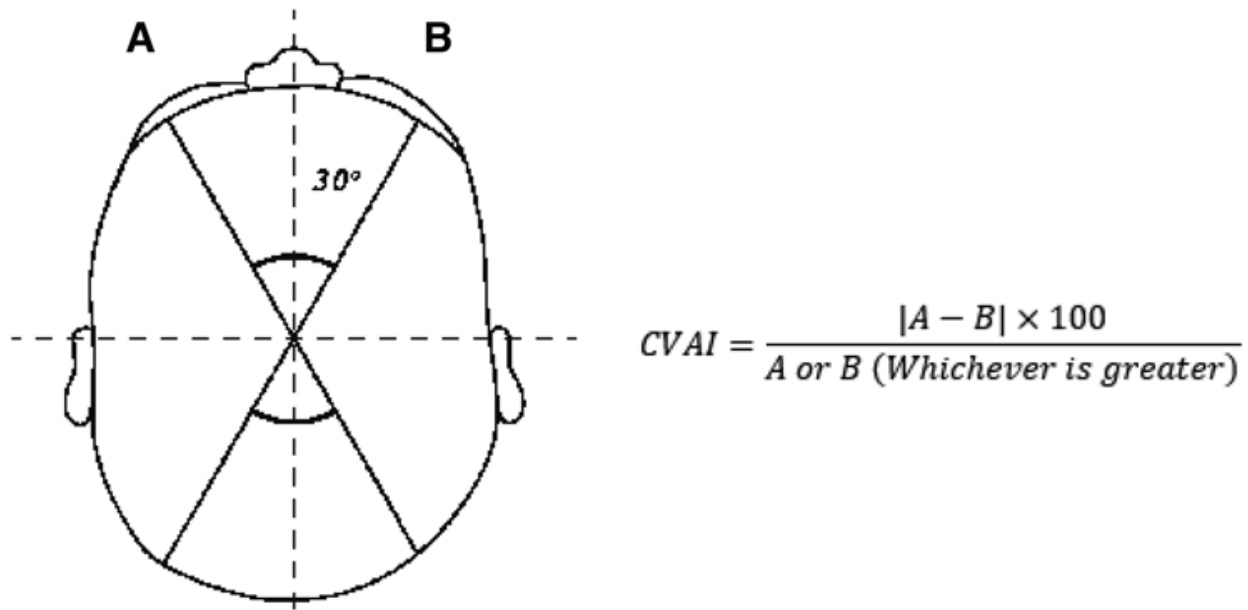

FIG. 1. Calculation of CVAI. ${ }^{7} \mathrm{~A}$ and $\mathrm{B}$ indicate the final locations of measurements used in the equation to calculate CVAl.

tional plagiocephaly. ${ }^{10}$ Multiple studies have attempted to determine the prevalence of plagiocephaly across various age groups; however, the results have been inconsistent. ${ }^{1,2,8}$

Our study, to our knowledge, is the largest to date utilizing the cranial vault asymmetry index (CVAI) and CT to determine the prevalence of PPP at a single tertiary care center. Using imaging from patients at Monroe Carell Jr. Children's Hospital at Vanderbilt (MCJCHV), we set out to evaluate the prevalence of PPP, whether it persists in the pediatric and adolescent population, and the change in prevalence with age. All patients included in this study were born after the prevailing adoption of the supine sleeping position recommended by the AAP in their Back to Sleep campaign, as well as the introduction of the orthotic helmet in 1998.

\section{Methods}

We conducted a cross-sectional study of pediatric patients evaluated for head trauma with a negative CT at MCJCHV between January 1 and December 31, 2018. The inclusion of study participants was based on the following criteria: 1) patients were age 18 years or younger at the time of CT imaging, 2) patients were evaluated at MCJCHV, and 3) CT imaging results were negative for posttraumatic focal or diffuse lesions at initial presentation. Exclusion of study patients was based on the following criteria: 1) history of shunted hydrocephalus, 2) radiographic presence of cranial trauma, and 3) history of craniosynostosis or skull surgery.

Patient data were retrospectively reviewed through electronic medical records at MCJCHV. The CVAI was assessed using CT imaging at the level of the superior orbital rim (Figs. 1 and 2). In accordance with previous literature, ${ }^{3}$ values for CVAI greater than $3.5 \%$ were evaluated as pathological asymmetry and were subcategorized as follows: mild (3.5\%-7\%), moderate $(7 \%-12 \%)$, and severe $(>12 \%)$. The patients were grouped by age to assess PPP at different stages of head development: group 1, 2-5 months (171 patients); group 2, 6-11 months (227 patients); group 3, 12-23 months (133 patients); group 4, 2-4 years (216 patients); group 5, 5-8 years (175 patients); group 6,
9-12 years (184 patients); and group 7, 13-18 years (323 patients). Many of the patients were evaluated only once for their trauma, and there was no longitudinal follow-up of individual patients.

\section{Results}

Overall, the total cohort included 1429 patients (611 [43\%] female and 818 [57\%] male) with a mean age of 6.6 years (95\% CI 6.25-6.9 years). Prevalence of PPP in the cohort of 1429 patients was $24.8 \%$ (354 patients). There was a consistent decline in PPP prevalence by age: group $1,40.4 \%(\mathrm{n}=171)$; group $2,33.5 \%(\mathrm{n}=227)$; group 3 , $33.8 \%(\mathrm{n}=133)$; group $4,26.4 \%(\mathrm{n}=216)$; group $5,20.0 \%$ $(\mathrm{n}=175)$; group $6,20.1 \%(\mathrm{n}=184)$; and group $7,10.8 \%$ $(\mathrm{n}=323)$. Mild cranial vault asymmetry was noted most often $(78.0 \%, 276$ patients), followed by moderate $(19.5 \%$, 69 patients) and severe asymmetry (2.5\%, 9 patients). Of the severe cases, $6(66.7 \%)$ were in patients younger than 6 months, with the remaining 3 patients identified in age groups 2, 3, and 5. There were no patients in group 6 or 7 with severe PPP. The prevalence and severity of PPP, subcategorized by age and CVAI score, are summarized in Table 1 and Fig. 3.

\section{Discussion}

The prevalence of plagiocephaly in the pediatric population has been a widely cited topic; ${ }^{3,8,13}$ however, the literature remains inconclusive on the exact number of children affected. The heterogenous nature of this discussion is partially attributable to the Back to Sleep campaign, founded by the AAP in 1992, which recommends that children sleep in the supine position. ${ }^{10}$ Additionally, the approval of orthotic helmet usage for plagiocephaly in 1998 offered another treatment option for these patients. ${ }^{12}$ The current large single-center retrospective study utilizing CT and the CVAI score ${ }^{3}$ to determine the prevalence of plagiocephaly across age groups is to our knowledge the largest to date. Our hypothesis is that the total prevalence of plagiocephaly decreases with increasing age of the child. The overall PPP prevalence was $24.8 \%$, with 

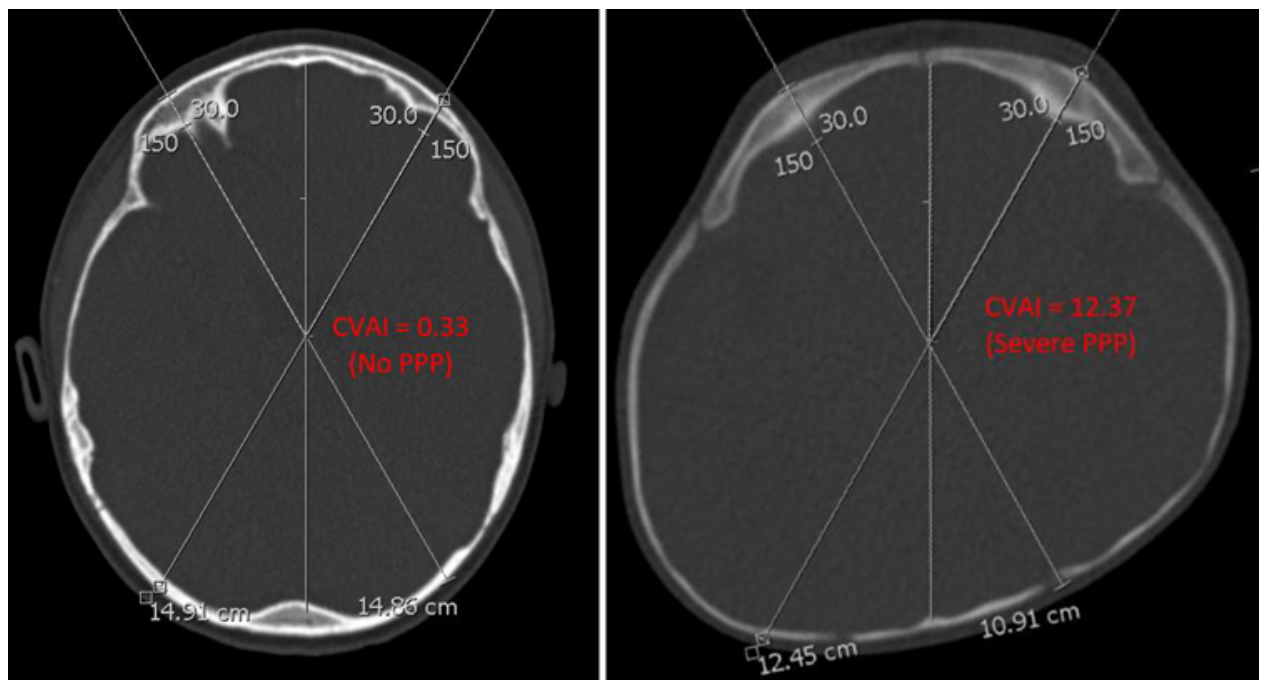

FIG. 2. CT images showing comparison of CVAI values and head shape in a patient without PPP and a patient with severe PPP. Figure is available in color online only.

prevalence and severity decreasing with increasing age. Moderate asymmetry was seen in all age groups, and no patients had severe deformity in adolescence.

Despite the patient populations affected by the Back to Sleep campaign since its inception in 1992, only a few notable studies have been published regarding plagiocephaly prevalence. Hutchison et al. ${ }^{8}$ conducted a prospective cohort study $(n=200)$ utilizing proprietary software and digital photography to determine the prevalence of nonsynostotic plagiocephaly at 6 weeks, 4 months, 8 months, 12 months, and 2 years. Their overall results demonstrated prevalences of $16 \%, 19.7 \%, 9.2 \%, 6.8 \%$, and $3.3 \%$ for each group, respectively, and substantiated a steady decline with increasing child age. An additional prospective longitudinal cohort study utilizing plagiocephalometry was conducted by van Vlimmeren et al..$^{13}$ in a cohort of 248 children, previously recruited,${ }^{14}$ to determine the incidence of PPP at 5 years of age. In $80 \%$ of patients, plagiocephaly was absent; mild plagiocephaly was identified in $19 \%$ of patients, and moderate to severe cases in $1 \%$ of patients. ${ }^{13}$
Most recently, and in contrast to the prevailing literature consensus, Di Rocco et al., ${ }^{3}$ via a retrospective analysis using a methodology similar to that used in our study, demonstrated persistence of moderate plagiocephaly in children ages $9-12$ and $13-18$ years $(n=165)$. Similarly to the results of the Di Rocco et al. study, our results showed a total prevalence of $24.8 \%$, and moderate asymmetry was seen in all age groups. The highest prevalence of severe plagiocephaly was noted in group 1 (age 2-5 months), which is when primarily supine positioning is recommended by the AAP Back to Sleep campaign.

This study was designed to determine the prevalence and natural history of PPP, regardless of treatment. As a result, a few important clinical features should be considered. First, major guideline changes, such as the AAP Back to Sleep positioning recommendations, can alter the prevalence of a disease, as in the case of PPP. Our findings show that 1 in 4 patients may potentially be affected by plagiocephaly to some degree. Given this high prevalence, parents should be warned about the possible development

TABLE 1. PPP distribution by age group and CVAI score

\begin{tabular}{ccccc}
\hline & \multicolumn{4}{c}{ PPP CVAI Score } \\
\cline { 2 - 5 } Age Group & Total & Mild & Moderate & Severe \\
\hline $1(n=171)$ & $69(40.4 \%)$ & $41(59.4 \%)$ & $22(31.9 \%)$ & $6(8.7 \%)$ \\
\hline $2(n=227)$ & $76(33.5 \%)$ & $52(68.4 \%)$ & $23(30.3 \%)$ & $1(1.3 \%)$ \\
\hline $3(n=133)$ & $45(33.8 \%)$ & $37(82.2 \%)$ & $7(15.6 \%)$ & $1(2.2 \%)$ \\
\hline $4(n=216)$ & $57(26.4 \%)$ & $48(84.4 \%)$ & $9(15.8 \%)$ & $0(0 \%)$ \\
\hline $5(n=175)$ & $35(20.0 \%)$ & $31(88.6 \%)$ & $3(8.6 \%)$ & $1(2.9 \%)$ \\
\hline $6(n=184)$ & $37(20.0 \%)$ & $36(97.3 \%)$ & $1(2.7 \%)$ & $0(0 \%)$ \\
\hline $7(n=323)$ & $35(10.8 \%)$ & $31(88.6 \%)$ & $4(11.4 \%)$ & $0(0 \%)$ \\
\hline All $(n=1429)$ & $354(24.8 \%)$ & $276(78.0 \%)$ & $69(19.5 \%)$ & $9(2.5 \%)$ \\
\hline
\end{tabular}

Values are presented as number (percentage) of patients in each age group (group 1, 2-5 months; group 2, 6-11 months; group 3, 12-23 months; group 4, 2-4 years; group 5, 5-8 years; group 6, 9-12 years; and group 7, 13-18 years). 


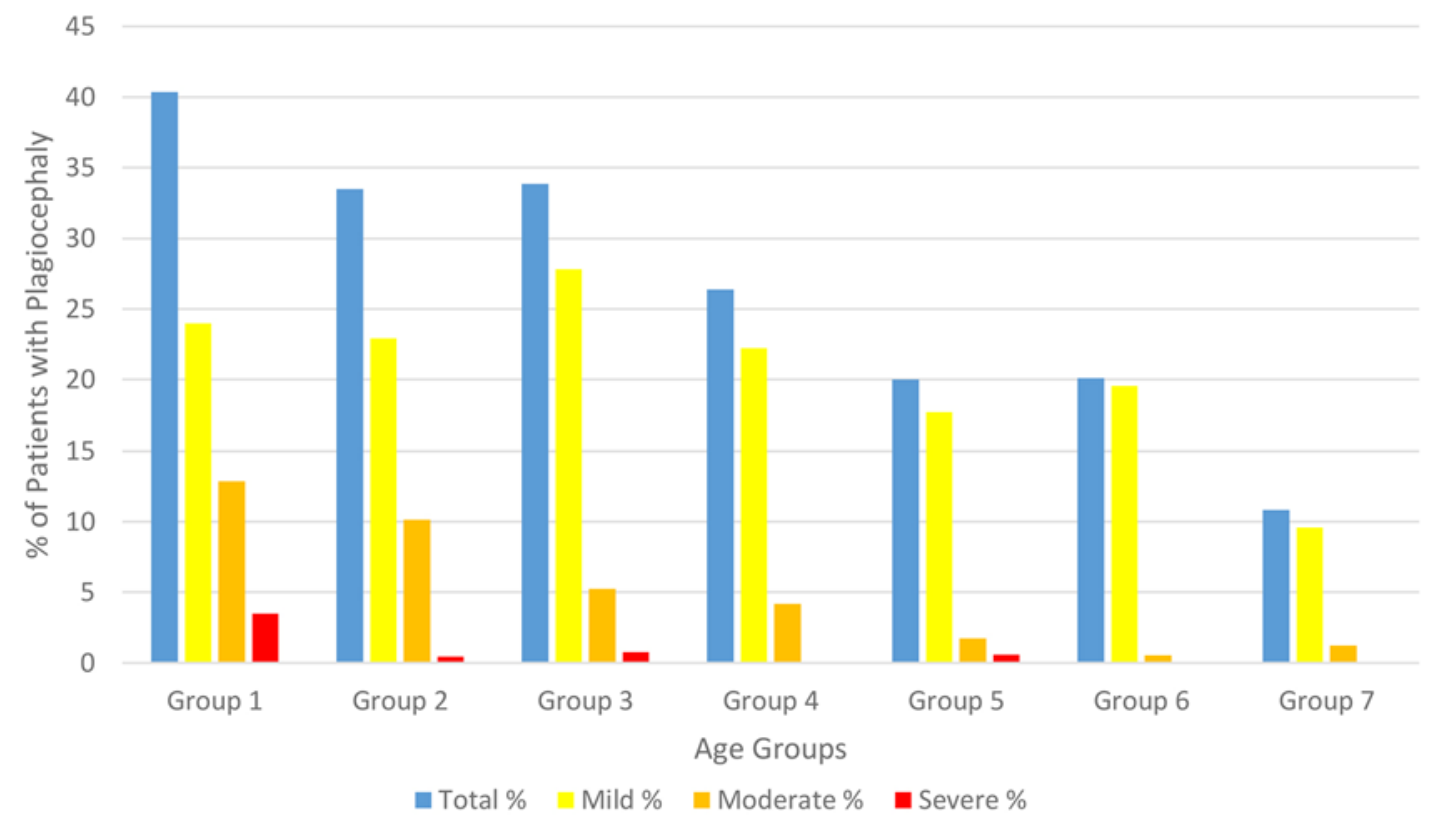

FIG. 3. Prevalence of plagiocephaly (PPP) by severity (CVAI score) across age groups (group 1, 2-5 months; group 2, 6-11 months; group 3, 12-23 months; group 4, 2-4 years; group 5, 5-8 years; group 6, 9-12 years; and group 7, 13-18 years). Figure is available in color online only.

of this benign condition as a result of placing children in the supine position as recommended in the AAP guidelines. This preemptive education can help alleviate excess worry, concern, or urgent appointments in busy neurosurgical clinics. Nonneurosurgical healthcare providers should also understand the natural history of plagiocephaly to help select patients for whom neurosurgical evaluation is needed. Additionally, if skull asymmetry is suspected, our study proves that it is most likely to be mild in nature, and that the total number of cases decrease with time. While our study did not take into consideration which patients ultimately underwent treatment for PPP, our results demonstrate that age has an effect on skull shape. In patients in age groups 4-7, who were beyond the accepted age of treatment, the total number of PPP cases consistently declined. This finding supports the understanding that PPP is likely to resolve over time and is particularly important when selecting patients for potential treatment.

\section{Study Limitations}

There are limitations to this study. First, the cranial measurements were made on CT images, and thus we did not have baseline aesthetic evaluations to see the clinical relevance of the skull asymmetry. Also, our study did not account for study patients who were currently or previously being managed for plagiocephaly and our data did not include the rate of past focused treatment or helmet use for PPP treatment. Thus, there is no way to accurately determine the rate of helmet treatment in our population, as many of the patients were evaluated only once for their trauma. Furthermore, there was no longitudinal follow-up of individual patients, which limited our ability to determine the evolution of PPP over time. Additionally, due to the epidemiological characteristics of trauma in pediat- ric patients, a majority of the images obtained were from male patients. Finally, this study does not include positional posterior brachycephaly, which is a symmetrical flattening of the occiput, ${ }^{4}$ and is measured by using the cranial index. ${ }^{6}$

\section{Conclusions}

PPP is prevalent in pediatric populations and most commonly presents as a case of mild asymmetry. Although there was an overall decline of PPP prevalence and severity with increasing patient age in our patients, moderate asymmetry was seen in all age groups. However, no patients in the cohort had severe asymmetry that persisted into adolescence. Early recognition and focused intervention may help prevent the persistence of skull asymmetry.

\section{References}

1. Biggs WS: Diagnosis and management of positional head deformity. Am Fam Physician 67:1953-1956, 2003

2. Collett BR: Helmet therapy for positional plagiocephaly and brachycephaly. BMJ 348:g2906, 2014

3. Di Rocco F, Ble V, Beuriat PA, Szathmari A, Lohkamp LN, Mottolese C: Prevalence and severity of positional plagiocephaly in children and adolescents. Acta Neurochir (Wien) 161:1095-1098, 2019

4. Feijen M, Franssen B, Vincken N, van der Hulst RR: Prevalence and consequences of positional plagiocephaly and brachycephaly. J Craniofac Surg 26:e770-e773, 2015

5. Graham T, Adams-Huet B, Gilbert N, Witthoff K, Gregory $\mathrm{T}$, Walsh M: Effects of initial age and severity on cranial remolding orthotic treatment for infants with deformational plagiocephaly. J Clin Med 8:E1097, 2019

6. Hinken L, Willenborg H, Dávila LA, Daentzer D: Outcome analysis of molding helmet therapy using a classification for differentiation between plagiocephaly, brachycephaly and 
combination of both. J Craniomaxillofac Surg 47:720-725, 2019

7. Holowka MA, Reisner A, Giavedoni B, Lombardo JR, Coulter C: Plagiocephaly severity scale to aid in clinical treatment recommendations. J Craniofac Surg 28:717-722, 2017

8. Hutchison BL, Hutchison LA, Thompson JM, Mitchell EA: Plagiocephaly and brachycephaly in the first two years of life: a prospective cohort study. Pediatrics 114:970-980, 2004

9. Korpilahti P, Saarinen P, Hukki J: Deficient language acquisition in children with single suture craniosynostosis and deformational posterior plagiocephaly. Childs Nerv Syst 28:419-425, 2012

10. Moon RY: SIDS and other sleep-related infant deaths: expansion of recommendations for a safe infant sleeping environment. Pediatrics 128:e1341-e1367, 2011

11. Panchal J, Amirsheybani H, Gurwitch R, Cook V, Francel P, Neas B, et al: Neurodevelopment in children with single-suture craniosynostosis and plagiocephaly without synostosis. Plast Reconstr Surg 108:1492-1500, 2001

12. Roby BB, Finkelstein M, Tibesar RJ, Sidman JD: Prevalence of positional plagiocephaly in teens born after the "Back to Sleep" campaign. Otolaryngol Head Neck Surg 146:823828,2012

13. van Vlimmeren LA, Engelbert RH, Pelsma M, Groenewoud HM, Boere-Boonekamp MM, der Sanden MW: The course of skull deformation from birth to 5 years of age: a prospective cohort study. Eur J Pediatr 176:11-21, 2017
14. van Vlimmeren LA, van der Graaf Y, Boere-Boonekamp MM, L'Hoir MP, Helders PJ, Engelbert RH: Risk factors for deformational plagiocephaly at birth and at 7 weeks of age: a prospective cohort study. Pediatrics 119:e408-e418, 2007

\section{Disclosures}

The authors report no conflict of interest concerning the materials or methods used in this study or the findings specified in this paper.

\section{Author Contributions}

Conception and design: Ahluwalia, Foster, Gannon, Wiseman, Shannon, Bonfield. Acquisition of data: all authors. Analysis and interpretation of data: all authors. Drafting the article: all authors. Critically revising the article: Ahluwalia, Foster, Shannon, Bonfield. Reviewed submitted version of manuscript: Ahluwalia, Foster, Shannon, Bonfield. Approved the final version of the manuscript on behalf of all authors: Ahluwalia. Statistical analysis: Ahluwalia, Foster, Shannon, Bonfield. Administrative/ technical/material support: Shannon, Bonfield. Study supervision: Ahluwalia, Shannon, Bonfield.

\section{Correspondence}

Ranbir Ahluwalia: Florida State University College of Medicine, Tallahassee,FL.ra16@med.fsu.edu. 